\title{
SO(10) unification with horizontal symmetry
}

\author{
Avik Banerjee $\odot,{ }^{1, *}$ Gautam Bhattacharyya $\odot,{ }^{1, \dagger}$ and Palash B. Pal $\odot^{2, *}$ \\ ${ }^{1}$ Saha Institute of Nuclear Physics, HBNI, 1/AF Bidhan Nagar, Kolkata 700064, India \\ ${ }^{2}$ Department of Physics, University of Calcutta, 92 APC Road, Kolkata 700009, India
}

(Received 13 May 2020; accepted 3 July 2020; published 20 July 2020)

\begin{abstract}
We extend the nonsupersymmetric $\mathrm{SO}(10)$ grand unification theories by adding a horizontal symmetry, which connects the three generations of fermions. Without committing to any specific symmetry group, we investigate the 1-loop renormalization group evolutions of the gauge couplings with one and two intermediate breaking scales. We find that depending on the $\mathrm{SO}(10)$ breaking chains, gauge coupling unification is compatible with only a handful of choices of representations of the Higgs bosons under the horizontal symmetry.
\end{abstract}

DOI: 10.1103/PhysRevD.102.015018

\section{INTRODUCTION}

The quest for the unification of fundamental forces remains to be a major motivation over the last few decades for investigating theories beyond the Standard Model. Although the smallest possible group, SU(5) [1], has been ruled out, the idea of unification is very much alive, resting on supersymmetric versions, and/or bigger unification groups like $\mathrm{SO}(10)$ [2-15]. In fact, $\mathrm{SO}(10)$ is the smallest unification group that contains all fermion fields of a single generation in one irreducible multiplet (irrep).

However, even a group like $\mathrm{SO}(10)$ cannot explain why there are three generations of fermions. Three copies of the irrep are obviously needed for three generations, though the number is theoretically arbitrary. Therefore, there is a lot of speculation whether an enhanced symmetry might shed some extra light on the number of fermion generations [16-20].

In this paper, we consider the possibility that the three fermion generations form a multiplet under some horizontal symmetry group $H$ that appears as a direct product with $\mathrm{SO}(10)$. In other words, the symmetry group of our model is $\mathrm{SO}(10) \times H$. We will examine the unification of the gauge coupling constants of the Standard Model (SM) into $\mathrm{SO}(10)$. In this pursuit, we do not need to assume anything specific about the nature of $H$. For example, we do not need to know whether $H$ is a global symmetry or a

\footnotetext{
*avik.banerjeesinp@saha.ac.in 'gautam.bhattacharyya@saha.ac.in "palashbaran.pal@saha.ac.in
}

Published by the American Physical Society under the terms of the Creative Commons Attribution 4.0 International license. Further distribution of this work must maintain attribution to the author(s) and the published article's title, journal citation, and DOI. Funded by SCOAP. gauge symmetry. Even more, we do not even need to specify whether it is a discrete group or a continuous group, or whether the three fermion generations comprise an irrep under this group. Multiplets of $H$ will only contribute in our analysis by providing multiple copies of the Higgs boson representations, which affect the evolution of the couplings of SM. Several earlier attempts have been made to use horizontal symmetries in explaining the flavor hierarchy in the fermion sector of the SM [21-37]. However, in most of those cases, the horizontal symmetry is imposed on the fermion mass matrix to generate the required texture. Despite these attempts, it is fair to say that the flavor problem has not yet been solved, and at the moment there is nothing close to what may be called the Standard Model of flavor physics. This necessitates a systematic investigation of all possible flavor avenues. In this paper, we rely on two basic working principles, namely, $S O(10)$ unification together with families, and admission of only renormalizable Yukawa operators. We will see that, without delving into the actual flavor issues, we could discriminate scenarios and disfavor quite a few of them based on our working assumptions. This of course narrows down the hunt for the yet elusive solution to the flavor problem.

The paper is organized as follows. In Sec. II, we outline the general features of the model, with a short description of SO(10) unification, followed by how the horizontal symmetry group works. In Sec. III, we introduce the various chains of symmetry breaking, indicating the Higgs boson representation whose vacuum expectation value (VEV) is responsible for each breaking, and the masses of the scalar bosons. In Sec. IV, we present all of our results for the symmetry breaking scales. These results show which chains are compatible with unification, and what would be the various scales of symmetry breaking in these chains. We end with some comments and outlook in Sec. V. 


\section{SO(10) WITH HORIZONTAL SYMMETRY}

In $\mathrm{SO}(10)$ grand unified theory (GUT), all left-chiral fermion and antifermion fields belonging to the same generation constitute a 16-dimensional irrep. All fermions obtain mass at the level of electroweak symmetry breaking. The only exception is the left-chiral antineutrino field (conjugate of the right-chiral neutrino field), which is a singlet under the SM gauge group, and can therefore obtain a Majorana mass at a higher level of symmetry breaking.

The model that we explore in this paper has a symmetry group $\mathrm{SO}(10) \times H$, where $H$ acts between generations. Our primary aim in this paper is to examine what multiplicity of various Higgs boson representations of $\mathrm{SO}(10)$ can be accommodated between different mass thresholds so that their contributions to the renormalization group (RG) evolution still lead to successful gauge unification. We draw reference to $H$ only to give credence to this multiplicity of Higgs bosons. Our conclusions would be based primarily on the number of appropriately light Higgs states in different stages of RG evolution, and not so much on what $H$ is and not at all on how exactly it breaks.

To explain this point, let us consider the couplings of the fermion multiplets with the scalars, which can be generically written as $\Psi \Psi \Phi$. Since the fermions of a single generation transform like a 16 representation of $\mathrm{SO}(10)$, the Higgs bosons coupling to the fermions must belong to the direct product $16 \times 16$ in order that the coupling can be a singlet of $\mathrm{SO}(10)$. In $\mathrm{SO}(10)$,

$$
16 \times 16=10+120+126,
$$

of which the first and the last irreps shown on the right side constitute symmetric combinations, while the 120 is antisymmetric. We will consider only the symmetric combinations, since the 120 is not necessary for conducting the gauge symmetry breaking of $\mathrm{SO}(10)$ down to the electroweak symmetry breaking level.

The three generations of fermions must transform like a 3-dimensional representation of the horizontal symmetry group. As already stated, it is not relevant for our analysis whether it is reducible or irreducible under $H$.

In writing the Yukawa couplings $\Psi \Psi \Phi$, we have omitted all indices. There are the Lorentz indices, the $\mathrm{SO}(10)$ indices, as well as the generation indices. The combination $\Psi \Psi$ should more explicitly be written as $\Psi^{\top} C \Psi$ where $C$ is a matrix, defined in such a way that $\Psi^{\top} C$ transforms like $\bar{\Psi}$ under Lorentz transformation. The combination $\Psi^{\top} C \Psi$ must be antisymmetric in the two $\Psi$ 's because the matrix $C$ is always antisymmetric, irrespective of the representation used for the Dirac matrices [38]. We have chosen to work with symmetric $\mathrm{SO}(10)$ combinations. Thus, according to the Pauli principle which dictates the overall exchange property to be antisymmetric with respect to the exchange of the two $\Psi$ 's, the combination $\Psi \Psi$ must be symmetric under the horizontal group.
This requirement does not uniquely determine the dimensionality of the scalar representations under the horizontal group. As we said earlier, we make no assumption regarding whether the three generations of fermions constitute an irrep of $H$. All we know, without specifying the horizontal group, is that the generations form a 3-dimensional representation, which can be irreducible or reducible. Specification of the horizontal group then dictates whether that 6-dimensional representation, obtained by taking the symmetric product of two 3-dimensional representations, is irreducible or reducible. Accordingly, the representation of $\Phi$ might be irreducible, or might be one of the irreps appearing in the product $\Psi \Psi$.

It is easy to see that different choices of the group $H$ can lead to all sorts of dimensions for the irreps. For example, if the horizontal symmetry group is $\mathrm{SU}(3)$, the fermion generations form a 3-dimensional irrep, and the symmetric product is a 6-dimensional irrep. If the symmetry group is $\mathrm{SO}(3)$ and the fermion generations form an irrep, the symmetric product contains a 5-dimensional and a 1-dimensional irrep. However, in this case there is also the possibility that the fermion generations do not form an irrep. If they transform as a $2+1$ dimensional representation, i.e., one 2-dimensional irrep and a 1-dimensional irrep, then the symmetric product includes 3,2 , and 1 dimensional irreps. In the discrete group $A_{4}$, the symmetric product of $3 \times 3$ contains a 3 -dimensional irrep, plus three different 1 dimensional irreps. If we choose $S_{3}$ as the horizontal group, which does not have a 3-dimensional irrep, the representation of the fermions can at best be $2+1$ dimensional. The symmetric product of such a reducible representation with itself contains both 2 and 1. In short, from symmetry consideration alone, we see anything from a 1-dimensional up to a 6-dimensional representation is allowed for $\Phi$.

If we use a $n$-dimensional representation for $\Phi$, with $1 \leq n \leq 6$, then there are $n$ copies of the $\mathrm{SO}(10)$ Higgs multiplets of that representation. These particles participate in the RG evolution above their mass thresholds, and affect gauge coupling unification. The demand of unification imposes what kind of representations are allowed for $\Phi$.

So far, we have been talking about Higgs multiplets which participate in Yukawa interaction. Symmetry breaking of the grand unified group $\mathrm{SO}(10)$ require other Higgs multiplets as well, as we will see in Sec. III. All other Higgs bosons, which do not couple to fermions, are assumed to be singlets of the horizontal group in our discussion.

\section{SO(10) BREAKING CHAINS}

$\mathrm{SO}(10)$ might break to the SM gauge group through only one intermediate group, i.e., the symmetry breaking chain may be of the form

$$
\begin{gathered}
\mathrm{SO}(10) \stackrel{M_{U}}{\longrightarrow} G_{1} \stackrel{M_{1}}{\longrightarrow} \mathrm{SU}(3)_{c} \times \mathrm{SU}(2)_{L} \times \mathrm{U}(1)_{Y} \\
\stackrel{M_{Z}}{\longrightarrow} \mathrm{SU}(3)_{c} \times \mathrm{U}(1)_{\mathrm{em}},
\end{gathered}
$$


TABLE I. Possible chains of $\mathrm{SO}(10)$ breaking with one intermediate scale. $G_{U}$ is the unification group. In the column SSB1, the number above the arrow sign gives the irrep of $\mathrm{SO}(10)$ whose VEV is responsible for the spontaneous symmetry breaking. The symbols below the arrows represent the multiplets of $G_{1}$ which contribute to the RG evolution as long as $G_{1}$ is unbroken. Breaking of $G_{1}$ is given in Eq. (3.3).

\begin{tabular}{|c|c|c|c|}
\hline Chain & $G_{U}$ & SSB1 & $G_{1}$ \\
\hline \multirow[t]{2}{*}{1} & \multirow[t]{2}{*}{$\mathrm{SO}(10)$} & $\stackrel{\langle 210\rangle}{\longrightarrow}$ & \multirow[t]{2}{*}{$2_{L} 2_{R} 4_{C}$} \\
\hline & & $\begin{array}{c}(2,2,1)_{10} \\
(1,3,10)_{126} /(1,2,4)_{16} \\
\langle 54\rangle\end{array}$ & \\
\hline 2 & $\mathrm{SO}(10)$ & $\begin{array}{c}\longrightarrow(2,2,1)_{10} \\
\left(\begin{array}{l}(1,3,10)_{126} /(1,2,4)_{16} \\
(3,1,10)_{126} /(2,1,4) 16\end{array}\right.\end{array}$ & $\left\{2{ }_{L} 2{ }_{R} 4_{C} P\right\}$ \\
\hline \multirow[t]{2}{*}{3} & \multirow{2}{*}{$\mathrm{SO}(10)$} & $\langle 45\rangle$ & \multirow{2}{*}{$2_{L} 2_{R} 1_{X} 3_{c}$} \\
\hline & & $\begin{array}{c}(2,2,0,1)_{10} \\
(1,3,1,1)\left(126 /\left(1,2, \frac{1}{2}, 1\right) 16\right. \\
\langle 210\rangle\end{array}$ & \\
\hline 4 & $\mathrm{SO}(10)$ & 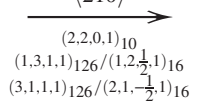 & $\left\{2_{L} 2_{R} 1_{X} 3_{c} P\right\}$ \\
\hline \multirow[t]{2}{*}{5} & \multirow{2}{*}{$\mathrm{SO}(10)$} & $\stackrel{\langle 45\rangle}{\longrightarrow}$ & \multirow{2}{*}{$2_{L} 1_{R} 4_{C}$} \\
\hline & & $\begin{array}{c}\left(2, \frac{1}{2}, 1\right)_{10} \\
(1,1,10)_{126} /\left(1,-\frac{1}{2}, 4\right)_{16} \\
\langle 210\rangle\end{array}$ & \\
\hline 6 & $\mathrm{SO}(10)$ & $\underset{\left.(1,1,-1,1), \frac{1}{2}, 0,1\right)_{12} /\left(1,-\frac{1}{2}, \frac{1}{2}, 1\right)_{16}}{\longrightarrow}$ & $\left\{2_{L} 1_{R} 1_{X} 3_{c}\right\}$ \\
\hline
\end{tabular}

where the symbols above the arrows represent the symmetry breaking scales. One possibility is that $G_{1}=\mathrm{SU}(5)$. We ignore this possibility, since then gauge coupling unification must happen at the $\mathrm{SU}(5)$ level, something that has been ruled out by precision data in nonsupersymmetric models. The other possible choices of $G_{1}$ have been shown in Table I.

From $\mathrm{SO}(10)$, the symmetry breaking can also go through two intermediate scales down to the SM gauge group, i.e., one can consider symmetry breaking chains like

$$
\begin{aligned}
& \mathrm{SO}(10) \stackrel{M_{U}}{\longrightarrow} G_{2} \stackrel{M_{2}}{\longrightarrow} G_{1} \stackrel{M_{1}}{\longrightarrow} \mathrm{SU}(3)_{c} \times \mathrm{SU}(2)_{L} \times \mathrm{U}(1)_{Y} \\
& \stackrel{M_{Z}}{\longrightarrow} \mathrm{SU}(3)_{c} \times \mathrm{U}(1)_{\mathrm{em}},
\end{aligned}
$$

with various choices for the intermediate groups $G_{1}$ and $G_{2}$. These possibilities have been shown in Table II. Breaking chains with more than two intermediate scales have been considered in the literature [39] as well, but we do not discuss them.

In writing Tables I and II as well as in subsequent discussions, we have adopted some shorthand notation used in many earlier articles on $\mathrm{SO}(10)$ breaking $[7,8,14]$. For the sake of completeness, we briefly explain the notations here.

(1) For example, let us look at the first chain given in Table I. It shows that the intermediate gauge group is $2{ }_{L}{ }_{R} 4_{C}$. This stands for $\mathrm{SU}(2)_{L} \times \mathrm{SU}(2)_{R} \times \mathrm{SU}(4)_{C}$, where the SU(4) factor stands for the Pati-Salam group [40] which treats leptons as a fourth "color," $\mathrm{SU}(2)_{L}$ belongs to the SM electroweak gauge group, and the other one is a right-handed SU(2) under which the right-chiral quark and lepton fields transform separately as doublets. Similarly, presence of the symbol $3_{c}$ would imply the SU(3) gauge group of QCD. The direct product factor $P$, which appears in some chains, is a discrete symmetry between the two SU(2) factors, which ensures that the coupling constants of the two SU(2)'s are equal.

(2) As for $1_{X}$ which is a $\mathrm{U}(1)$ subgroup, the quantum numbers shown in Tables I and II equal to $(B-L) / 2$. With this definition, the normalization of $X$ does not agree with that of the non-Abelian factors, so the quantum numbers will have to be multiplied by a factor of $\sqrt{3 / 2}$ in order to be used in $\mathrm{RG}$ equations. For the $1_{R}$ subgroup however, we put the eigenvalues of the corresponding generator of the $2_{R}$ subgroup, which have the proper normalization.

(3) In Table I, the column bearing the heading "SSB1" contains information about symmetry breaking and masses of the Higgs bosons. The number above the arrow gives the $\mathrm{SO}(10)$ representation which has a neutral component whose VEV can perform the desired symmetry breaking. Of course these multiplets have VEVs at the unification scale, and therefore all their components are expected to have masses at the unifications scale, meaning that they do not affect the RG equations. But, in the regime below the $\mathrm{SO}(10)$ breaking scale where the group $G_{1}$ is the unbroken gauge group, the RG equations contain contributions from some Higgs boson submultiplets which contain VEVs that affect one of the lower stages of symmetry breaking. They have been shown below the arrow, indicating their transformations under the unbroken group at that stage, and marked with a subscript that tells us which $\mathrm{SO}(10)$ representation contains them. The rationale for choosing the masses of the Higgs bosons will be described in Sec. IV.

(4) We have not shown in Table I which multiplet of SO (10) breaks the intermediated symmetry down to the SM symmetry group, and further trigger the electroweak symmetry breaking. This is because this part is the same for all chains, and is given by

$$
G_{1} \underset{\left(2, \frac{1}{2}, 1\right)_{10}}{\stackrel{\langle 126 / 16\rangle}{\longrightarrow}} 2_{L} 1_{Y} 3_{c} \stackrel{\langle 10\rangle}{\longrightarrow} 1_{Q} 3_{c} .
$$

As seen here, we have considered two alternatives for breaking $G_{1}$ to the SM gauge group, separated by a slash in Eq. (3.3). One is by the 126-dimensional representation of $\mathrm{SO}(10)$, which has Yukawa coupling with fermions. The other is by using the 
TABLE II. Possible chains of $\mathrm{SO}(10)$ breaking with two intermediate scales.

\begin{tabular}{|c|c|c|c|c|c|}
\hline Chain & $G_{U}$ & SSB2 & $G_{2}$ & SSB1 & $G_{1}$ \\
\hline \multirow{2}{*}{ I } & \multirow{2}{*}{ SO(10) } & $\langle 210\rangle$ & \multirow{2}{*}{$2_{L} 2_{R} 4_{C}$} & $\langle 45\rangle$ & \multirow{2}{*}{$2_{L} 2_{R} 1_{X} 3_{c}$} \\
\hline & & $\begin{array}{c}(2,2,1)_{10} \\
\longrightarrow \\
(1,3,10)_{126} /(1,2,4) 16 \\
(1,1,15)_{45}\end{array}$ & & $\underset{(1,3,-1,1)_{126} /\left(1,2, \frac{1}{2}, 1\right)_{16}}{\longrightarrow}$ & \\
\hline \multirow[b]{2}{*}{ II } & \multirow[b]{2}{*}{$\mathrm{SO}(10)$} & $\langle 54\rangle$ & \multirow[b]{2}{*}{$2_{L} 2_{R} 4_{C} P$} & $\langle 210\rangle$ & \multirow[b]{2}{*}{$2_{L} 2_{R} 1_{X} 3_{c} P$} \\
\hline & & 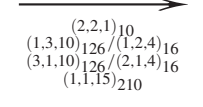 & & $\begin{array}{c}\underset{(2,2,2,1)_{10}}{\longrightarrow} \\
(1,3,-1,1)_{126} /\left(1,2, \frac{1}{2}, 1\right)_{16} \\
(3,1,-1,1)_{126} /\left(2,1, \frac{1}{2}, 1\right)_{16}\end{array}$ & \\
\hline \multirow[b]{2}{*}{ III } & \multirow[b]{2}{*}{$\mathrm{SO}(10)$} & $\stackrel{\langle 54\rangle}{\longrightarrow}$ & \multirow[b]{2}{*}{$2_{L} 2_{R} 4_{C} P$} & $\stackrel{\langle 45\rangle}{\longrightarrow}$ & \multirow[b]{2}{*}{$2_{L} 2_{R} 1_{X} 3_{c}$} \\
\hline & & 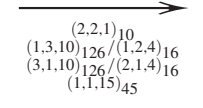 & & $\underset{\left(1,3,-1,1,1,126 /\left(1,2, \frac{1}{2}, 1\right)\right.}{\longrightarrow}$ & \\
\hline \multirow[b]{2}{*}{ IV } & \multirow[b]{2}{*}{$\mathrm{SO}(10)$} & $\langle 210\rangle$ & \multirow[b]{2}{*}{$2_{L} 2_{R} 1_{X} 3_{c} P$} & $\langle 45\rangle$ & \multirow[b]{2}{*}{$2_{L} 2_{R} 1_{X} 3_{c}$} \\
\hline & & $\begin{array}{c}\underset{(2,2,0,1)_{10}}{\longrightarrow} \\
\left(\begin{array}{ll}(1,3,-1,1) \\
(3,1,1,1,1) /\left(1,2, \frac{1}{1}, 1\right)_{16} \\
\left(1,1,\left(2,1,-\frac{1}{2}, 1\right)_{16}\right.\end{array}\right.\end{array}$ & & $\underset{(1,3,-1,1)_{126} /\left(1,2, \frac{1}{2}, 1\right)_{16}}{\longrightarrow}$ & \\
\hline \multirow{2}{*}{ V } & \multirow{2}{*}{$\mathrm{SO}(10)$} & $\stackrel{\langle 210\rangle}{\longrightarrow}$ & \multirow{2}{*}{$2_{L} 2_{R} 4_{C}$} & $\stackrel{\langle 45\rangle}{\longrightarrow}$ & \multirow{2}{*}{$2_{L} 1_{R} 4_{C}$} \\
\hline & & $\begin{array}{c}(2,2,1) 10 \\
(1,3,10)(12) /(1,2,4)_{16} \\
(1,3,1)_{45}\end{array}$ & & $\begin{array}{c}\left(2, \frac{1}{2}, 1\right)_{10} \\
(1,1,10)_{126} /\left(1,-\frac{1}{2}, 4\right)_{16}\end{array}$ & \\
\hline \multirow[b]{2}{*}{ VI } & \multirow[b]{2}{*}{$\mathrm{SO}(10)$} & $\langle 54\rangle$ & \multirow[b]{2}{*}{$2_{L} 2_{R} 4_{C} P$} & $\langle 45\rangle$ & \multirow[b]{2}{*}{$2_{L} 1_{R} 4_{C}$} \\
\hline & & $\begin{array}{c}\longrightarrow(2,2,1) 10 \\
\longrightarrow(1,3,10)_{126} /(1,2,4)_{16} \\
(3,1,10)_{126} /(2,1,4)_{16} \\
(1,3,1)_{45}+(3,1,1)_{45}\end{array}$ & & $\underset{\left(2, \frac{1}{2}, 1\right)_{10}}{\longrightarrow}$ & \\
\hline \multirow[b]{2}{*}{ VII } & \multirow{2}{*}{$\mathrm{SO}(10)$} & $\stackrel{\langle 54\rangle}{\longrightarrow}$ & \multirow[b]{2}{*}{$2_{L} 2_{R} 4_{C} P$} & $\langle 210\rangle$ & \multirow{2}{*}{$2_{L} 2_{R} 4_{C}$} \\
\hline & & 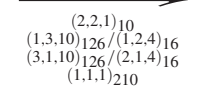 & & $\frac{(2,2,1)_{10}}{(1,3,10)_{126} /(1,2,4)_{16}}$ & \\
\hline \multirow{3}{*}{ VIII } & \multirow{2}{*}{$\mathrm{SO}(10)$} & $\stackrel{\langle 45\rangle}{\longrightarrow}$ & \multirow{2}{*}{$2_{L} 2_{R} 1_{X} 3_{c}$} & $\stackrel{\langle 45\rangle}{\longrightarrow}$ & \multirow{2}{*}{$2_{L} 1_{R} 1_{X} 3_{c}$} \\
\hline & & $\begin{array}{c}(2,2,0,1)_{10} \\
(1,3,-1,1)\left(126 /\left(1,2, \frac{1}{2}, 1\right)_{16}\right. \\
(1,3,0,1)_{45}\end{array}$ & & $\underset{\left(2, \frac{1}{2}, 0,1\right)_{10}}{\longrightarrow}$ & \\
\hline & & $\langle 210\rangle$ & & $\langle 45\rangle$ & \\
\hline IX & $\mathrm{SO}(10)$ & $\begin{array}{c}(2,2,0,1)_{10} \\
(1,3,-1,1)_{126} /\left(1,2, \frac{1}{2}, 1\right)_{16} \\
(3,1,1,1)_{126} /\left(2,1,-\frac{1}{2}, 1\right)_{16} \\
(1,3,0,1)_{45}+(3,1,0,1)_{45}\end{array}$ & $\left\{2_{L} 2_{R} 1_{X} 3_{c} P\right\}$ & $\begin{array}{c}\left(2, \frac{1}{2}, 0,1\right)_{10} \\
(1,1,-1,1)_{126} /\left(1,-\frac{1}{2}, \frac{1}{2}, 1\right)_{16}\end{array}$ & $\left\{2_{L} 1_{R} 1_{X} 3_{c}\right\}$ \\
\hline \multirow{2}{*}{$X$} & \multirow{2}{*}{$\mathrm{SO}(10)$} & $\underset{\langle 210\rangle}{\longrightarrow}$ & $\left\{2{ }_{I}{ } 4{ }_{C}\right\}$ & $\langle 210\rangle$ & \\
\hline & & $\begin{array}{c}(2,2,1)_{10} \\
(1,3,10)_{126 /(1,2,4)_{16}} \\
(1,3,15)_{210}\end{array}$ & $\left\{Z_{L} Z_{R}{ }^{4} C\right\}$ & $\begin{array}{l}\left(2, \frac{1}{2}, 0,1\right)_{10} \\
(1,1,-1,1) 1_{126} /\left(1,-\frac{1}{2}, \frac{1}{2}, 1\right)_{16}\end{array}$ & $\left\{L_{L}{ }^{1} R^{1} X^{3} c\right\}$ \\
\hline & & $\langle 54\rangle$ & & $\langle 210\rangle$ & \\
\hline XI & $\mathrm{SO}(10)$ & 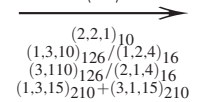 & $\left\{2{ }_{L} 2{ }_{R} 4_{C} P\right\}$ & $\underset{\substack{\left(2, \frac{1}{2}, 0,1\right) \\
(1,1,-1,1)_{126} /\left(1,-\frac{1}{2}, \frac{1}{2}, 1\right)}}{\longrightarrow}$ & $\left\{2_{L} 1_{R} 1_{X} 3_{c}\right\}$ \\
\hline & & $\stackrel{\langle 45\rangle}{\longrightarrow}$ & & $\stackrel{\langle 45\rangle}{\longrightarrow}$ & \\
\hline XII & $\mathrm{SO}(10)$ & 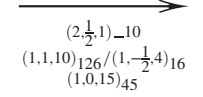 & $\left\{2_{L} 1_{R} 4_{C}\right\}$ & $\underset{\left(2, \frac{1}{2}, 0,1\right) 10}{\longrightarrow}$ & $\left\{2_{L} 1_{R} 1_{X} 3_{c}\right\}$ \\
\hline
\end{tabular}

16-dimensional irrep, which does not have Yukawa coupling at the tree-level, but can contribute to fermion masses through loops [41]. In subsequent tables and discussions, for the first alternative, the chain is specified by adding the letter "a," whereas the letter " $b$ " is added for the second alternative.

(5) The same notations are used for chains with two intermediate scales. The only difference is that, in this case, one needs to specify the Higgs boson multiplets which perform the intermediate scale symmetry breaking, $G_{2} \rightarrow G_{1}$. Thus, the column with the heading "SSB2" contains information about the Higgs multiplet that performs the breaking $\mathrm{SO}(10) \rightarrow G_{2}$, and the submultiplets of $G_{2}$ which we consider for the RG equations above the $G_{2^{-}}$ breaking scale. Similarly, the column marked "SSB1" contains information about the multiplet of $\mathrm{SO}(10)$ that is responsible for the breaking $G_{2} \rightarrow G_{1}$, and the submultiplets of $G_{1}$ which are assumed to be light above the $G_{1}$-breaking scale. 


\section{RESULTS}

\section{A. Outline of the strategy}

The 1-loop RG evolution of the gauge coupling $g$ for an $\mathrm{SU}(N)$ factor above the weak scale is governed by the equation

$$
\frac{d \omega}{d \ln \mu}=\frac{1}{2 \pi}\left(\frac{11}{3} N-4-\frac{T(S)}{6}\right),
$$

where

$$
\omega=\frac{4 \pi}{g^{2}},
$$

and $T(S)$ is the scalar contribution. For a U(1) factor in the symmetry group, one should take $N=0$ in Eq. (4.1). Note that, in writing the fermion contribution in Eq. (4.1), we assumed for simplicity that symmetry breaking scales are heavier than the masses of all fermions which obtain masses at that stage of breaking. In fact, we consider the right-handed neutrinos to have Majorana masses which are smaller than $M_{1}$, the scale at which all symmetries specific to the right-handed fermions break down in the chain of symmetry breaking from $\mathrm{SO}(10)$, i.e., below which the SM group appears.

For the scalar contribution, we need to have some idea of the Higgs boson masses. As is usual practice in this kind of analysis, we use the extended survival hypothesis $[42,43]$ to estimate the masses. This means that, at any stage of symmetry breaking, the entire submultiplet containing the VEV obtains mass at that scale, and any particle not controlled by this rule obtains mass at the unification scale. We have further extended this hypothesis across generations, i.e., whatever is the mass hierarchy generated due to $\mathrm{SO}(10)$ breaking for one family is replicated for other (at least one more) families so as to generate Higgs multiplicities. We have already listed, in Tables I and II, the Higgs boson submultiplets which contribute to $T(S)$ in all different regimes. The overall contributions to $T(S)$ for all intermediate symmetry groups have been listed in earlier literature $[7,8]$ where only one 10-dimensional and one 126-dimensional SO(10) multiplets of Higgs boson were considered. For the present purpose, all we need is to multiply the contributions of 10 and 126 irreps by the appropriate number of multiplets.

So we start with the values of the gauge coupling constants of the SM gauge group at the $Z$-scale [44]:

$$
\begin{gathered}
\omega_{1_{\mathrm{Y}}}\left(M_{Z}\right)=59.042 \pm 0.003, \\
\omega_{2_{\mathrm{L}}}\left(M_{Z}\right)=29.596 \pm 0.005, \\
\omega_{3_{\mathrm{c}}}\left(M_{Z}\right)=8.47 \pm 0.02 .
\end{gathered}
$$

As has already been said, the evolution depends on the chain of symmetry breaking, for which we use the nomenclature used by earlier authors and repeated here in Tables I and II. In addition, the evolution depends on the numbers of 10 and 126 irreps of Higgs bosons used, which we will denote by $r_{10}$ and $r_{126}$ respectively. For each chain, and each choice of $r_{10}$ and $r_{126}$, we employ the following checks to determine whether a given chain is allowed.

(1) There must be a solution for all gauge couplings meeting at a scale.

(2) When a mathematical solution is obtained, it must be physically meaningful. For example, in Eq. (3.2), if $\mathrm{SO}(10)$ breaks at $M_{U}$, whereas $G_{2}$ and $G_{1}$ break at the scales $M_{2}$ and $M_{1}$ respectively, one must have

$$
n_{U}>n_{2}>n_{1}>n_{Z}
$$

where the $n_{i}$ 's represent a logarithmic notation that we use for the various energy scales:

$$
n_{i}=\log _{10}\left(\frac{M_{i}}{1 \mathrm{GeV}}\right)
$$

(3) Further, one must have $M_{U} \lesssim 10^{18} \mathrm{GeV}$ because gravity effects become strong at higher scales, and the analysis that ignores gravity makes no sense.

(4) The unification scale must be consistent with proton decay bound. The lifetime of proton in terms of the GUT scale and couplings can be written as

$$
\tau_{p} \simeq \frac{\omega_{U}^{2} M_{U}^{4}}{m_{p}^{5}},
$$

where $m_{p}$ denotes proton mass. Present limit on the proton lifetime is [44]

$$
\tau_{p}\left(p \rightarrow e^{+} \pi^{0}\right)>1.6 \times 10^{34} \mathrm{yr} .
$$

However, taking a cue from [14], we have made an order of magnitude estimate to accommodate the higher-loop effects in the proton decay bound. Our estimate shows that proton lifetime as calculated using 1-loop results for the GUT parameters can be enhanced by two orders of magnitude if 2-loop effects are incorporated. In view of this conservative estimation, we also allow those chains which satisfy

$$
\tau_{p}\left(p \rightarrow e^{+} \pi^{0}\right)>1.6 \times 10^{32} \mathrm{yr} .
$$

We clearly mark which chains satisfy Eq. (4.7) and which ones satisfy only this relaxed bound, Eq. (4.8). 
(5) All couplings are consistent with the perturbative limit

$$
\omega>\frac{1}{4 \pi} \text {. }
$$

If any one of these conditions is not satisfied for a chain, the chain is ruled out, and its details are not given in the tables.

In our calculations, whenever it is relevant, we take kinetic mixing of $\mathrm{U}(1)$ gauge factors into account, something that was not done in the early papers on the subject but was incorporated in the later papers [14,45-47]. Our results for the allowed chains, and the allowed values of various scales of symmetry breaking, are presented in the rest of this section. For the sake of convenience, we divide the discussion into two parts: one in which there is only one intermediate scale, and the other in which there are two such scales.

\section{B. One intermediate scale}

If there is only one intermediate scale, the possibilities of the intermediate gauge group have been shown in Table I. The results obtained from the 1-loop RG equations have

TABLE III. Results for chains with a-type symmetry breaking with one intermediate scale: intermediate scale $\left(n_{1}\right)$, unification scale $\left(n_{U}\right)$ and unification coupling $\left(\omega_{U}\right)$ have been shown for each case where we find an acceptable solution. Nomenclatures for the chains can be read off from Table I. A down arrow to the right of the value of $n_{U}$ indicates that the given combination of unification scale and coupling does not satisfy the proton lifetime bound in Eq. (4.7), but allowed by the conservative bound in Eq. (4.8).

\begin{tabular}{|c|c|c|c|c|c|}
\hline \multirow[b]{2}{*}{$\left(r_{10}, r_{126}\right)$} & \multirow[b]{2}{*}{ Quantity } & \multicolumn{4}{|c|}{ Breaking chains } \\
\hline & & $1 \mathrm{a}$ & $2 \mathrm{a}$ & $3 a$ & $4 a$ \\
\hline \multirow[t]{3}{*}{$(1,1)$} & $n_{1}$ & 11.75 & 13.71 & 9.02 & 10.11 \\
\hline & $n_{U}$ & 16.06 & $15.22 \downarrow$ & 16.66 & 15.77 \\
\hline & $\omega_{U}$ & 45.70 & 41.20 & 46.19 & 43.89 \\
\hline \multirow[t]{3}{*}{$(1,2)$} & $n_{1}$ & & & 6.16 & \\
\hline & $n_{U}$ & & & 16.54 & \\
\hline & $\omega_{U}$ & & & 45.89 & \\
\hline \multirow[t]{3}{*}{$(2,1)$} & $n_{1}$ & 12.19 & & 10.24 & 10.95 \\
\hline & $n_{U}$ & $15.53 \downarrow$ & & 15.90 & $15.29 \downarrow$ \\
\hline & $\omega_{U}$ & 44.11 & & 44.23 & 42.66 \\
\hline \multirow[t]{3}{*}{$(2,2)$} & $n_{1}$ & & & 8.28 & \\
\hline & $n_{U}$ & & & 15.75 & \\
\hline & $\omega_{U}$ & & & 43.84 & \\
\hline \multirow[t]{3}{*}{$(2,3)$} & $n_{1}$ & & & 4.49 & \\
\hline & $n_{U}$ & & & $15.46 \downarrow$ & \\
\hline & $\omega_{U}$ & & & 43.09 & \\
\hline \multirow[t]{3}{*}{$(3,1)$} & $n_{1}$ & & & 11.19 & \\
\hline & $n_{U}$ & & & $15.30 \downarrow$ & \\
\hline & $\omega_{U}$ & & & 42.69 & \\
\hline
\end{tabular}

been summarized in two tables, where we show the intermediate scale, the unification scale and the unification coupling for all chains which admit an acceptable solution. In Table III, we consider the SM symmetry group to appear through the breaking using 126 multiplet of Higgs bosons, whereas in Table IV, we consider the possibility that the said intermediate symmetry breaking occurs through a 16 multiplet of Higgs bosons. If any chain does not appear in these tables, or there is no entry for the scales corresponding to any chain, it means that there is no solution consistent with the criteria outlined earlier.

For all chains, the case with $r_{10}=r_{126}=1$ takes us to the limiting scenario with no horizontal symmetry. The differences of our results with that obtained earlier [7] using 126-irrep are because of two reasons. First, we use updated inputs for the couplings at the weak scale, given in Eq. (4.3) and for the constraint on proton lifetime. Second, while we have performed only a 1-loop calculation here, 2-loop results are available in the literature [7]. However, note that we keep some room for the 2-loop effects using some simple estimates as mentioned earlier.

We now highlight the new results found in the present paper. First, in a-type chains, we analyze the effects of the values of $r_{10}$ and $r_{126}$ larger than 1 , which are necessary for discussing horizontal symmetry. Second, cases for b-type symmetry breaking $[48,49]$, which use a 16 instead of a 126 for breaking down to the level of the SM symmetry group, have been analyzed here with $r_{10}>1$.

Note that we obtain no solution at all for chains 5 and 6 in Table I. This means that, if there is only one intermediate stage between the GUT group and the SM group, then that intermediate stage must contain the full $\mathrm{SU}(2)_{R}$ symmetry.

If we look at models where at least one of $r_{10}$ and $r_{126}$ is bigger than 1, we see that chain 2 is also ruled out, and chain $1 \mathrm{a}$ is allowed only when $r_{10}=2$. This means that the full $\mathrm{SU}(4)_{C}$ symmetry at the intermediate stage is disfavored, or equivalently that the $\mathrm{SU}(3)_{c}$ group of QCD should appear right at the grand unified symmetry breaking.

It is also seen that there is no solution in any symmetry breaking chain when either $r_{10}$ and $r_{126}$ is bigger than 3 . The intermediate group $2_{L} 2_{R} 1_{X} 3_{c}$ seems to be most suitable, in the sense that it is most flexible with the numbers of the Higgs boson multiplets. Its companion version with the extra parity symmetry does equally well for b-type chains, but not so well with the a-type chains.

\section{Two intermediate scales}

We now turn to the symmetry breaking chains which involve two intermediate stages. As is well known, it is not possible to uniquely solve the values of the various breaking scales in this case. Since there are only three boundary conditions corresponding to the experimentally measured values of the SM gauge couplings at the weak scale, while there are four unknown variables, namely $n_{1}$, 
TABLE IV. Results for chains with b-type symmetry breaking with one intermediate scale. Notations are same as in Table III.

\begin{tabular}{lccccc}
\hline \hline & & \multicolumn{4}{c}{ Breaking chains } \\
\cline { 3 - 6 }$\left(r_{10}\right)$ & Quantity & $1 \mathrm{~b}$ & $2 \mathrm{~b}$ & $3 \mathrm{~b}$ & $4 \mathrm{~b}$ \\
\hline$(1)$ & $n_{1}$ & 13.63 & 13.71 & 10.41 & 10.67 \\
& $n_{U}$ & $15.39 \downarrow$ & $15.35 \downarrow$ & 16.72 & 16.49 \\
& $\omega_{U}$ & 45.07 & 44.64 & 46.34 & 45.75 \\
$(2)$ & $n_{1}$ & & & 11.23 & 11.40 \\
& $n_{U}$ & & & 15.97 & 15.82 \\
& $\omega_{U}$ & & & 44.42 & 44.02 \\
$(3)$ & $n_{1}$ & & & 11.89 & 12.00 \\
& $n_{U}$ & & & $15.37 \downarrow$ & $15.27 \downarrow$ \\
& $\omega_{U}$ & & & 42.89 & 42.62 \\
\hline \hline
\end{tabular}

$n_{2}, n_{U}$ and $\omega_{U}$, one can at most find some regions allowed by the selection criteria for each of them. All solutions which pass these tests have been listed. Table V contain results for a-type breaking chains, whereas Table VI contain results for b-type breaking chains. Since the number of possible chains is huge, we had to break each of these tables into two parts for convenience of display, first giving results for chains I to V, and next for chains VI to X. For chains XI and XII, we find no solution, so they do not appear in the tables.

In the results presented in Tables $\mathrm{V}$ and VI, different quantities given on the left sides of the dashes as a set, correspond to one solution, whereas those of the right sides correspond to another solution. Thus, for example, with chain Ia and $\left(r_{10}, r_{126}\right)=(1,1)$, one extreme of the solution range lies at $n_{1}=9.02$ and $n_{2}=n_{U}=16.66$, whereas the other extreme solution is at $n_{1}=11.17$,

TABLE V. The range of allowed values for a-type symmetry breaking with two intermediate scales. Nomenclatures for the chains can be read off from Table II. In case of $n_{U}$, an arrow on either side of the separator points to the combination of unification scale and coupling that does not satisfy the proton lifetime bound in Eq. (4.7), but allowed by the conservative bound in Eq. (4.8).

\begin{tabular}{|c|c|c|c|c|c|c|}
\hline \multicolumn{7}{|c|}{ Chains Ia to Va } \\
\hline$\left(r_{10}, r_{126}\right)$ & Quantity & Ia & IIa & IIIa & IVa & $\mathrm{Va}$ \\
\hline$(1,1)$ & $\begin{array}{l}n_{1} \\
n_{2} \\
n_{U} \\
\omega_{U}\end{array}$ & $\begin{array}{c}9.02-11.17 \\
16.66-11.19 \\
16.66-16.75 \\
46.19-46.42\end{array}$ & $\begin{array}{c}10.12-13.70 \\
15.77-13.71 \\
15.77 \rightarrow 15.35 \\
43.89-41.03\end{array}$ & $\begin{array}{c}9.02-13.70 \\
16.66-13.72 \\
16.66 \rightarrow 15.36 \\
46.19-41.03\end{array}$ & $\begin{array}{c}9.02-10.11 \\
16.65-10.15 \\
16.66-15.77 \\
46.19-43.91\end{array}$ & $\begin{array}{c}11.36-11.58 \\
13.63-11.66 \\
15.10 \leftarrow 16.00 \\
44.75-45.62\end{array}$ \\
\hline$(1,2)$ & $\begin{array}{l}n_{1} \\
n_{2} \\
n_{U} \\
\omega_{U}\end{array}$ & $\begin{array}{c}2.00-6.15 \\
15.94-16.54 \\
17.67-16.54 \\
46.87-45.89\end{array}$ & $\begin{array}{c}12.66-13.71 \\
14.02-13.71 \\
15.14 \leftrightarrow 15.22 \\
38.59-37.51\end{array}$ & $\begin{array}{c}6.16-13.71 \\
16.54-13.71 \\
16.54 \rightarrow 15.22 \\
45.88-37.51\end{array}$ & $\begin{array}{c}6.16-9.17 \\
16.54-10.49 \\
16.54 \rightarrow 15.13 \\
45.88-42.25\end{array}$ & \\
\hline$(1,3)$ & $\begin{array}{l}n_{1} \\
n_{2} \\
n_{U} \\
\omega_{U}\end{array}$ & & & $\begin{array}{c}2.00-13.08 \\
15.93-13.83 \\
16.12 \rightarrow 15.16 \\
43.72-35.01\end{array}$ & $\begin{array}{c}2.00-5.06 \\
14.78-12.38 \\
15.84 \rightarrow 15.12 \\
44.08-42.24\end{array}$ & \\
\hline$(2,1)$ & $\begin{array}{l}n_{1} \\
n_{2} \\
n_{U} \\
\omega_{U}\end{array}$ & $\begin{array}{l}10.24-11.79 \\
15.89-11.79 \\
15.90-16.02 \\
44.23-44.53\end{array}$ & $\begin{array}{c}10.95-12.72 \\
5.29-14.28 \\
15.29 \leftrightarrow 15.13 \\
42.66-41.32\end{array}$ & $\begin{array}{c}10.24-13.34 \\
15.90-13.94 \\
15.90 \rightarrow 15.13 \\
44.22-40.96\end{array}$ & $\begin{array}{c}10.24-10.95 \\
15.87-10.98 \\
15.89 \rightarrow 15.29 \\
44.22-42.67\end{array}$ & $\begin{array}{c}11.91-12.07 \\
12.99-12.09 \\
15.12 \leftrightarrow 15.50 \\
43.80-44.07\end{array}$ \\
\hline$(2,2)$ & $\begin{array}{l}n_{1} \\
n_{2} \\
n_{U} \\
\omega_{U}\end{array}$ & $\begin{array}{c}2.00-8.28 \\
14.34-15.75 \\
17.35-15.75 \\
44.64-43.84\end{array}$ & & $\begin{array}{c}2.00-8.28 \\
14.34-15.75 \\
17.35-15.75 \\
44.64-43.84\end{array}$ & $\begin{array}{c}8.28-9.55 \\
15.74-12.78 \\
15.75 \rightarrow 15.12 \\
43.84-42.24\end{array}$ & \\
\hline$(2,3)$ & $\begin{array}{l}n_{1} \\
n_{2} \\
n_{U} \\
\omega_{U}\end{array}$ & $\begin{array}{c}2.00-4.49 \\
15.39-15.46 \\
15.72 \rightarrow 15.46 \\
43.05-43.09\end{array}$ & & $\begin{array}{c}4.49-9.28 \\
15.45-14.55 \\
15.45 \leftrightarrow 15.14 \\
43.09-39.04\end{array}$ & $\begin{array}{c}4.49-5.84 \\
15.45-14.18 \\
15.45 \leftrightarrow 15.12 \\
43.09-42.24\end{array}$ & \\
\hline$(3,1)$ & $\begin{array}{l}n_{1} \\
n_{2} \\
n_{U} \\
\omega_{U}\end{array}$ & $\begin{array}{c}11.19-12.29 \\
15.30-12.30 \\
15.30 \leftrightarrow 15.42 \\
42.69-43.00\end{array}$ & & $\begin{array}{c}11.19-11.99 \\
15.30-14.80 \\
15.30 \leftrightarrow 15.13 \\
42.69-41.88\end{array}$ & $\begin{array}{c}11.19-11.38 \\
15.30-13.75 \\
15.30 \leftrightarrow 15.12 \\
42.69-42.25\end{array}$ & \\
\hline
\end{tabular}


TABLE V. (Continued)

\begin{tabular}{|c|c|c|c|c|c|c|}
\hline \multicolumn{7}{|c|}{ Chains Ia to Va } \\
\hline$\left(r_{10}, r_{126}\right)$ & Quantity & Ia & IIa & IIIa & $\mathrm{IVa}$ & $\mathrm{Va}$ \\
\hline$(3,2)$ & $\begin{array}{l}n_{1} \\
n_{2} \\
n_{U} \\
\omega_{U}\end{array}$ & $\begin{array}{c}2.00-9.85 \\
12.80-15.16 \\
17.04 \rightarrow 15.16 \\
42.50-42.33\end{array}$ & & $\begin{array}{c}9.86-10.11 \\
15.16-15.06 \\
15.16 \leftrightarrow 15.12 \\
42.32-42.04\end{array}$ & $\begin{array}{c}9.86-9.92 \\
15.15-14.99 \\
15.16 \leftrightarrow 15.13 \\
42.32-42.25\end{array}$ & \\
\hline$(3,3)$ & $\begin{array}{l}n_{1} \\
n_{2} \\
n_{U} \\
\omega_{U}\end{array}$ & $\begin{array}{c}2.00-4.95 \\
14.56-14.74 \\
15.40 \leftrightarrow 15.13 \\
41.10-41.40\end{array}$ & & & & \\
\hline$(4,2)$ & $\begin{array}{l}n_{1} \\
n_{2} \\
n_{U} \\
\omega_{U}\end{array}$ & $\begin{array}{c}2.00-9.17 \\
11.32-13.99 \\
16.75 \rightarrow 15.13 \\
40.44-41.01\end{array}$ & & & & \\
\hline$(5,2)$ & $\begin{array}{l}n_{1} \\
n_{2} \\
n_{U} \\
\omega_{U}\end{array}$ & $\begin{array}{c}2.00-8.26 \\
9.90-12.67 \\
16.46 \rightarrow 15.14 \\
38.47-39.57\end{array}$ & & & & \\
\hline$(6,2)$ & $\begin{array}{l}n_{1} \\
n_{2} \\
n_{U} \\
\omega_{U}\end{array}$ & $\begin{array}{c}2.00-7.26 \\
8.54-11.22 \\
16.19 \rightarrow 15.15 \\
36.56-37.98\end{array}$ & & & & \\
\hline \multicolumn{7}{|c|}{ Chains VIa to Xa } \\
\hline$\left(r_{10}, r_{126}\right)$ & Quantity & VIa & VIIa & VIIIa & IXa & $\mathrm{Xa}$ \\
\hline$(1,1)$ & $\begin{array}{l}n_{1} \\
n_{2} \\
n_{U} \\
\omega_{U}\end{array}$ & $\begin{array}{c}13.55-13.71 \\
13.77-13.71 \\
15.13 \leftrightarrow 15.16 \\
41.31-41.11\end{array}$ & $\begin{array}{c}11.75-13.71 \\
16.06-13.71 \\
16.06 \leftrightarrow 15.22 \\
45.69-41.21\end{array}$ & $\begin{array}{c}2.00-8.57 \\
7.89-8.58 \\
16.62-16.64 \\
46.07-46.14\end{array}$ & $\begin{array}{c}2.00-10.58 \\
9.88-10.58 \\
15.34 \leftrightarrow 15.50 \\
42.79-43.21\end{array}$ & \\
\hline$(1,2)$ & $\begin{array}{l}n_{1} \\
n_{2} \\
n_{U} \\
\omega_{U}\end{array}$ & & $\begin{array}{c}2.00-13.59 \\
14.88-13.72 \\
18.88 \rightarrow 15.15 \\
28.65-37.84\end{array}$ & $\begin{array}{c}2.00-5.31 \\
4.51-5.31 \\
16.48-16.51 \\
45.71-45.80\end{array}$ & & \\
\hline$(1,3)$ & $\begin{array}{l}n_{1} \\
n_{2} \\
n_{U} \\
\omega_{U}\end{array}$ & & $\begin{array}{c}3.57-12.99 \\
11.01-13.52 \\
17.36 \rightarrow 15.18 \\
0.08-32.63\end{array}$ & & & \\
\hline$(1,4)$ & $\begin{array}{l}n_{1} \\
n_{2} \\
n_{U} \\
\omega_{U}\end{array}$ & & $\begin{array}{c}8.54-11.23 \\
11.38-12.60 \\
15.71 \leftrightarrow 15.30 \\
2.81-18.36\end{array}$ & & & \\
\hline$(2,1)$ & $\begin{array}{l}n_{1} \\
n_{2} \\
n_{U} \\
\omega_{U}\end{array}$ & & $\begin{array}{c}12.19-13.23 \\
15.53-14.29 \\
15.53 \leftrightarrow 15.13 \\
44.11-41.80\end{array}$ & $\begin{array}{c}2.00-9.93 \\
9.14-9.93 \\
15.81-15.87 \\
44.01-44.17\end{array}$ & & \\
\hline$(2,2)$ & $\begin{array}{l}n_{1} \\
n_{2} \\
n_{U} \\
\omega_{U}\end{array}$ & & $\begin{array}{c}2.00-12.68 \\
14.88-13.81 \\
18.28 \rightarrow 15.15 \\
28.95-37.26\end{array}$ & $\begin{array}{c}2.00-7.73 \\
6.42-7.73 \\
15.60 \leftarrow 15.70 \\
43.47-43.73\end{array}$ & & \\
\hline
\end{tabular}


TABLE V. (Continued)

\begin{tabular}{|c|c|c|c|c|c|c|}
\hline \multicolumn{7}{|c|}{ Chains VIa to Xa } \\
\hline$\left(r_{10}, r_{126}\right)$ & Quantity & VIa & VIIa & VIIIa & IXa & $\mathrm{Xa}$ \\
\hline \multirow[t]{4}{*}{$(2,3)$} & $n_{1}$ & & 3.19-11.61 & $2.00-3.26$ & & \\
\hline & $n_{2}$ & & $10.90-13.15$ & 2.75-3.26 & & \\
\hline & $n_{U}$ & & $16.95 \leftrightarrow 15.21$ & $15.32 \leftrightarrow 15.36$ & & \\
\hline & $\omega_{U}$ & & 0.09-28.64 & $42.75-42.85$ & & \\
\hline \multirow[t]{4}{*}{$(3,1)$} & $n_{1}$ & & & $2.00-10.97$ & & \\
\hline & $n_{2}$ & & & $10.12-10.97$ & & \\
\hline & $n_{U}$ & & & $15.18 \leftrightarrow 15.28$ & & \\
\hline & $\omega_{U}$ & & & $42.40-42.63$ & & \\
\hline \multirow[t]{4}{*}{$(3,2)$} & $n_{1}$ & & $2.00-11.65$ & & & \\
\hline & $n_{2}$ & & $14.88-13.92$ & & & \\
\hline & $n_{U}$ & & $17.72 \rightarrow 15.16$ & & & \\
\hline & $\omega_{U}$ & & $29.22-36.60$ & & & \\
\hline \multirow[t]{4}{*}{$(3,3)$} & $n_{1}$ & & 2.81-9.91 & & & \\
\hline & $n_{2}$ & & $10.80-12.70$ & & & \\
\hline & $n_{U}$ & & $16.55 \leftrightarrow 15.25$ & & & \\
\hline & $\omega_{U}$ & & $0.08-23.70$ & & & \\
\hline \multirow[t]{4}{*}{$(4,2)$} & $n_{1}$ & & $2.00-10.47$ & & & \\
\hline & $n_{2}$ & & $14.88-14.03$ & & & \\
\hline & $n_{U}$ & & $17.19 \rightarrow 15.16$ & & & \\
\hline & $\omega_{U}$ & & $29.48-35.85$ & & & \\
\hline \multirow[t]{4}{*}{$(4,3)$} & $n_{1}$ & & $2.53-7.69$ & & & \\
\hline & $n_{2}$ & & 10.73-12.11 & & & \\
\hline & $n_{U}$ & & $16.16 \leftrightarrow 15.32$ & & & \\
\hline & $\omega_{U}$ & & $0.36-17.23$ & & & \\
\hline \multirow[t]{4}{*}{$(5,1)$} & $n_{1}$ & & & & & $2.00-4.71$ \\
\hline & $n_{2}$ & & & & & 3.89-4.71 \\
\hline & $n_{U}$ & & & & & $16.13-16.03$ \\
\hline & $\omega_{U}$ & & & & & $38.84-39.03$ \\
\hline \multirow[t]{4}{*}{$(5,2)$} & $n_{1}$ & & 2.00-9.11 & & & \\
\hline & $n_{2}$ & & $14.88-14.17$ & & & \\
\hline & $n_{U}$ & & $16.70 \rightarrow 15.17$ & & & \\
\hline & $\omega_{U}$ & & $29.72-34.99$ & & & \\
\hline \multirow[t]{4}{*}{$(6,2)$} & $n_{1}$ & & $2.00-7.53$ & & & \\
\hline & $n_{2}$ & & $14.88-14.33$ & & & \\
\hline & $n_{U}$ & & $16.24 \rightarrow 15.17$ & & & \\
\hline & $\omega_{U}$ & & 29.94-33.98 & & & \\
\hline
\end{tabular}

$n_{2}=11.19$ and $n_{U}=16.75$. Of course, all intermediate values are allowed. Since the RG equations are linear in the scales and also in $\omega_{U}$, the values of $n_{2}, n_{U}$, and $\omega_{U}$ corresponding to any intermediate value of $n_{1}$ can be obtained by linear interpolation.

As for the cases with single intermediate stage, the solutions with $r_{10}=r_{126}=1$ merely reproduce the solutions obtained in the absence of $H$. Our results agree roughly with the results in earlier 1-loop calculations [8], except for chain XIIa which is ruled out anyway. Slight deviations are due to reasons mentioned in the context of symmetry breaking with one intermediate scale. Also, in chains VIII to XII where there are two U(1) factors, we take kinetic mixing into account.

\section{DISCUSSIONS}

Here we briefly outline some important aspects and limitations of this work.

(1) It has to be understood that our results are based on 1-loop calculations only. It is known that higherloop calculation and threshold corrections may sometimes produce large changes in the RG solutions $[14,50]$. To leave room for the effect of 2-loop 
TABLE VI. Results for b-type symmetry breaking with two intermediate scales. Notation for the separators have been explained in the caption of Table V.

\begin{tabular}{|c|c|c|c|c|c|c|}
\hline \multicolumn{7}{|c|}{ Chains $\mathrm{Ib}$ to $\mathrm{Vb}$} \\
\hline$\left(r_{10}\right)$ & Quantity & $\mathrm{Ib}$ & IIb & IIIb & $\mathrm{IVb}$ & $\mathrm{Vb}$ \\
\hline (1) & $\begin{array}{l}n_{1} \\
n_{2} \\
n_{U} \\
\omega_{U}\end{array}$ & $\begin{array}{c}10.41-13.62 \\
16.72-13.62 \\
16.72 \rightarrow 15.56 \\
46.34-45.26\end{array}$ & $\begin{array}{c}10.67-13.71 \\
16.49-13.71 \\
16.49 \rightarrow 15.51 \\
45.74-44.77\end{array}$ & $\begin{array}{c}10.41-13.71 \\
16.72-13.71 \\
16.72 \rightarrow 15.51 \\
46.34-44.77\end{array}$ & $\begin{array}{c}10.41-13.71 \\
16.72-13.71 \\
16.72 \rightarrow 15.51 \\
46.34-44.77\end{array}$ & $\begin{array}{c}13.05-13.59 \\
14.20-13.59 \\
15.11 \leftrightarrow 15.37 \\
44.80-45.05\end{array}$ \\
\hline (2) & $\begin{array}{l}n_{1} \\
n_{2} \\
n_{U} \\
\omega_{U}\end{array}$ & $\begin{array}{c}11.23-13.64 \\
15.97-13.64 \\
15.97 \rightarrow 15.20 \\
44.42-43.96\end{array}$ & $\begin{array}{c}11.41-13.71 \\
15.81-13.71 \\
15.82 \rightarrow 15.16 \\
44.02-43.58\end{array}$ & $\begin{array}{c}11.23-13.71 \\
15.97-13.71 \\
15.97 \rightarrow 15.16 \\
44.42-43.58\end{array}$ & $\begin{array}{l}11.23-11.40 \\
15.93-11.42 \\
15.97-15.82 \\
44.42-44.02\end{array}$ & \\
\hline (3) & $\begin{array}{l}n_{1} \\
n_{2} \\
n_{U} \\
\omega_{U}\end{array}$ & $\begin{array}{c}11.89-12.77 \\
15.37-14.51 \\
15.37 \leftrightarrow 15.12 \\
42.89-42.83\end{array}$ & $\begin{array}{c}12.01-12.59 \\
15.27-14.73 \\
15.27 \leftrightarrow 15.12 \\
42.62-42.57\end{array}$ & $\begin{array}{c}11.89-12.75 \\
15.37-14.59 \\
15.37 \leftrightarrow 15.12 \\
42.89-42.70\end{array}$ & $\begin{array}{c}11.89-12.00 \\
15.27-12.03 \\
15.37 \leftrightarrow 15.27 \\
42.88-42.62\end{array}$ & \\
\hline \multicolumn{7}{|c|}{ Chains VIb to $\mathrm{Xb}$} \\
\hline$\left(r_{10}\right)$ & Quantity & $\mathrm{VIb}$ & VIIb & VIIIb & $\mathrm{IXb}$ & $\mathrm{Xb}$ \\
\hline (1) & $\begin{array}{l}n_{1} \\
n_{2} \\
n_{U} \\
\omega_{U}\end{array}$ & $\begin{array}{c}13.24-13.71 \\
14.14-13.71 \\
15.11 \leftrightarrow 15.28 \\
44.45-44.39\end{array}$ & $\begin{array}{c}13.63-13.71 \\
15.32-13.78 \\
15.39 \leftrightarrow 15.35 \\
45.05-44.65\end{array}$ & $\begin{array}{c}2.00-10.11 \\
9.92-10.11 \\
16.70-16.71 \\
46.29-46.31\end{array}$ & $\begin{array}{c}2.00-11.07 \\
10.89-11.07 \\
16.09-16.12 \\
44.72-44.79\end{array}$ & $\begin{array}{c}2.00-12.45 \\
12.29-12.45 \\
15.67-15.72 \\
45.30-45.36\end{array}$ \\
\hline (2) & $\begin{array}{l}n_{1} \\
n_{2} \\
n_{U} \\
\omega_{U}\end{array}$ & & & $\begin{array}{c}2.00-11.01 \\
10.81-11.01 \\
15.94-15.96 \\
44.34-44.38\end{array}$ & $\begin{array}{c}2.00-11.68 \\
11.49-11.68 \\
15.53 \leftrightarrow 15.56 \\
43.28-43.37\end{array}$ & $\begin{array}{c}2.00-12.71 \\
12.55-12.71 \\
15.25 \leftrightarrow 15.29 \\
43.87-43.94\end{array}$ \\
\hline (3) & $\begin{array}{l}n_{1} \\
n_{2} \\
n_{U} \\
\omega_{U}\end{array}$ & & & $\begin{array}{c}2.00-11.73 \\
11.52-11.73 \\
15.34 \leftrightarrow 15.36 \\
42.79-42.84\end{array}$ & & \\
\hline
\end{tabular}

corrections we somewhat relaxed the proton decay bound using an order of magnitude estimation. We emphasize that 2-loop estimation and 1-loop threshold corrections carry additional baggage of uncertainties due to the involvement of more parameters arising from specific details of model spectra. All these effectively relax the proton decay bound, which constitutes the basis of our strategy.

(2) Since the Higgs fields are charged under $H$, their VEVs indeed break $H$. In fact, $H$ might have already been broken by the VEV of an $\mathrm{SO}(10)$ singlet at an even higher scale. The question is whether all Higgs masses are pushed up to that scale by a naïve application of the extended survival hypothesis. We must remember that this hypothesis is at best a guess that one applies in the absence of knowledge of the detailed Higgs potential guided by symmetries. In this paper, whenever we refer to some $H$, we assume that multiplicities, arising due to $H$, of a given $n$-plet of $\mathrm{SO}(10)$ are treated identically in the potential. Then, since a 10-plet is lighter than the 126-plet as a consequence of $\mathrm{SO}(10)$ breaking sequence, all copies 10-plets are lighter than all copies of 126-plets. This is our extended survival hypothesis for the present analysis. Note, we remained agnostic about $H$ throughout the paper. We neither mentioned if $H$ breaks to nothing or to some subgroup, nor did we attempt to write down a full potential involving $H$ as a symmetry. The latter would have been horrifically complicated and severely model dependent. Even if one is not ready to accept our hypothesis, our analysis still goes through, as our primary objective is to examine how many light copies of $\mathrm{SO}(10) n$-plets can be kicked into life to contribute to RG evolution at different stages, regardless of what $H$ is. The extreme opposite scenario that all copies, except a lone generation of $\mathrm{SO}(10)$, become super-heavy at the topmost scale where $H$ breaks, however, deprecates any multiplicity of states, as if there is no 
horizontal symmetry, thus trivializing our analysis. However, this is only a limiting scenario.

(3) From our 1-loop results, one trend is quite clear: too many copies of light Higgs multiplets is not good for a model. For a-type symmetry breaking, employing 126-plets leading to renormalizable Yukawa interactions, more than three copies of the 10-plet or the 126-plet works only for very few chains. For b-type symmetry breaking, which utilizes 16-plets of Higgs bosons instead of 126 , we obtain results for at most three copies of the 10-plet.

(4) In some of the symmetry breaking chains, the $U(1)_{B-L}$ factor appears explicitly in the intermediate stages. In some others, it is part of an SU(4) factor. For the first set of chains, our results show that, if $r_{10}=r_{126}=1$, most of these chains yield a large value of $M_{1} \sim 10^{9} \mathrm{GeV}$, except chains VIIIa and IXa predicting much lower $M_{1}$ around $\mathrm{TeV}$ scale. However, for $r_{126}>1$, from Tables III and V we observe that the $\mathrm{U}(1)_{\mathrm{B}-\mathrm{L}}$ breaking scale $M_{1}$ can be brought to much lower values, within the reach of collider experiments. The presence of a $\mathrm{TeV}$ scale Abelian gauge boson $\left(Z^{\prime}\right)$ in those cases may have testable signatures in various flavor and electroweak observables. A detailed discussion is beyond the mandate of this paper.

(5) To make any speculation and draw conclusion on $H$ we need to make further assumption that the splitting pattern among Higgs masses is controlled purely by the pattern of $\mathrm{SO}(10)$ breaking sequence. For example, consider $\mathrm{SO}(3)$ as the horizontal symmetry group. For $\mathrm{SO}(3)$, the symmetric combination of $3 \times$ 3 contains a 5-plet and a singlet. We can conclude that $\mathrm{SO}(3)$ is disfavored if all the 5 copies of the 10-plet remain light till $M_{Z}$, since Table $\mathrm{V}$ shows $r_{10}=5$ necessarily requires $r_{126}=2$. The other escape route is to consider two copies of 126-dimensional Higgs which are singlet under $\mathrm{SO}(3)$. If, however, the potential is such that some horizontal copies receive much higher masses, thereby reducing the multiplicity contributing to RG in intermediate stages, then our previous observation of $\mathrm{SO}(3)$ being disfavored gets accordingly diluted. Analogous conclusions can also be drawn for other choices of $H$, e.g., $\mathrm{SU}(3)$.

(6) It is of course not necessary to think in terms of irreps only. If, for example, we need 4 copies of the 10, they need not form a 4-dimensional irrep of $H$. One can have a triplet and a singlet, or maybe two doublets. All we need is that they are part of the symmetric product of two triplets of $H$, since we are dealing with three generations. Alternatively, the fermions can also live in reducible representations under $H$. For example, if the horizontal symmetry is $S_{3}$, the SM quarks and leptons can transform as $2+1$, where the third generation fermions form the singlet. This might be useful in order to fulfill the phenomenological requirement of reproducing the correct flavor hierarchy.

Additional SO(10) Higgs multiplets, other than 10- and 126-plets, might also be advocated to meet some phenomenological criteria in the context of flavor physics, which can affect the unification of the gauge couplings. Here, we consider the minimal possibility from the perspective of grand unification only.

(7) A few comments on the issue of predictability of these models are in order. In the absence of any horizontal symmetry, any $\mathrm{SO}(10)$ predictions relating fermion masses must be confined within the same family, because the couplings of different generations would be independent. Horizontal symmetry provides an option for interfamily predictions. Note that if we put all the three families in a single irrep, the number of Yukawa couplings reduces dramatically rendering the model superpredictable. This is too restrictive and already falsified by data. On the contrary, if we put the fermion and/or scalar families in reducible representations having multiple irreps, as stressed earlier, the tension eases a bit. For example, the triplet 3 representation of the discrete group $S_{3}$ is a reducible representation: $3=2+1$. If we put the first two fermion families in the doublet of $S_{3}$, and the third family as the singlet, the observed heaviness of the third generation can be accounted for, while leaving the splitting between the first two families to additional model building. In other words, while the intention behind imposing horizontal symmetry is to reduce the number of parameters to increase predictability, real world data compel us to take shelter under the cover of multiple irreps at the expense of introducing a few more parameters. Though we lose some predictability this way, nevertheless, the number of such parameters never exceeds or even touches the corresponding number for conventional three-family $\mathrm{SO}(10)$ without any family symmetry at all. It is this issue that underscores the need for a horizontal symmetry.

(8) It is not just a question of fermion masses. There are other issues in flavor physics, like fermion mixing and $C P$ violation. For them as well, multiple irreps might prove essential. For example, in the context of nonunified models multiple Higgs doublets motivated by some horizontal symmetry, have been employed in the literature to deduce the general nature of the CKM matrix [51-54]. Various models predicting the masses and mixing in the neutrino sector also utilize more than one Higgs multiplets with varying degree of success. Similarly in the GUT models, existence of reducible representations of $H$ would definitely be an added advantage to 
predict the observed flavor texture. Moreover an enriched scalar sector may predict novel phenomenological signatures, which are otherwise unavailable. For example, more copies can give additional freedom of accommodating $C P$ violation at low scale.

(9) Values of $r_{10}$ larger than 1 imply multiple Higgs doublets with masses around the weak scale. Such scenarios may receive severe constraints from flavor changing neutral current processes, from the direct searches of charged Higgs bosons, etc. Thus our selection criteria can, in principle, confront the presence of multi-Higgs models at the weak scale.

(10) In this paper we work with three generations of chiral fermion, though our analysis can be easily extended to accommodate more generations. We have observed that for four chiral generations, charged under $H$, even fewer chains of symmetry breaking would be allowed.

(11) As a final comment, we mention that our analysis can also be extended to GUT models based on $E_{6}$ and larger groups as well [16-20], which might constitute an interesting exercise.

\section{ACKNOWLEDGMENTS}

A. B. acknowledges discussions with Triparno Bandyopadhyay, and support from the Department of Atomic Energy, Government of India. G. B. acknowledges support of the J.C. Bose National Fellowship from the Department of Science and Technology, Government of India (SERB Grant No. SB/S2/JCB-062/2016). The work of P. B. P. was supported by the SERB Grant No. EMR/ 2017/001434 of the Government of India.
[1] H. Georgi and S. L. Glashow, Unity of All Elementary Particle Forces, Phys. Rev. Lett. 32, 438 (1974).

[2] H. Georgi, The state of the art-Gauge theories, AIP Conf. Proc. 23, 575 (1975).

[3] H. Fritzsch and P. Minkowski, Unified interactions of leptons and hadrons, Ann. Phys. (N.Y.) 93, 193 (1975).

[4] R. Slansky, Group theory for unified model building, Phys. Rep. 79, 1 (1981).

[5] C. S. Aulakh and R. N. Mohapatra, Implications of supersymmetric SO(10) grand unification, Phys. Rev. D 28, 217 (1983).

[6] K. S. Babu and E. Ma, Symmetry breaking in SO(10): Higgs boson structure, Phys. Rev. D 31, 2316 (1985).

[7] N. G. Deshpande, E. Keith, and P. B. Pal, Implications of LEP results for $\mathrm{SO}(10)$ grand unification, Phys. Rev. D 46, 2261 (1993).

[8] N. G. Deshpande, E. Keith, and P. B. Pal, Implications of LEP results for $\mathrm{SO}(10)$ grand unification with two intermediate stages, Phys. Rev. D 47, 2892 (1993).

[9] C. S. Aulakh and A. Girdhar, $\mathrm{SO}(10)$ a la Pati-Salam, Int. J. Mod. Phys. A 20, 865 (2005).

[10] C. S. Aulakh, B. Bajc, A. Melfo, G. Senjanovic, and F. Vissani, The minimal supersymmetric grand unified theory, Phys. Lett. B 588, 196 (2004).

[11] T. Fukuyama, A. Ilakovac, T. Kikuchi, S. Meljanac, and N. Okada, $\mathrm{SO}(10)$ group theory for the unified model building, J. Math. Phys. (N.Y.) 46, 033505 (2005).

[12] K. S. Babu, I. Gogoladze, P. Nath, and R. M. Syed, A unified framework for symmetry breaking in $\mathrm{SO}(10)$, Phys. Rev. D 72, 095011 (2005).

[13] S. Bertolini, T. Schwetz, and M. Malinsky, Fermion masses and mixings in $\mathrm{SO}(10)$ models and the neutrino challenge to SUSY GUTs, Phys. Rev. D 73, 115012 (2006).

[14] S. Bertolini, L. Di Luzio, and M. Malinsky, Intermediate mass scales in the non-supersymmetric $\mathrm{SO}(10)$ grand unification: A reappraisal, Phys. Rev. D 80, 015013 (2009).

[15] D. Croon, T. E. Gonzalo, L. Graf, N. Košnik, and G. White, GUT physics in the era of the LHC, Front. Phys. 7, 76 (2019).

[16] J. E. Camargo-Molina, A. P. Morais, R. Pasechnik, and J. Wessén, On a radiative origin of the Standard Model from trinification, J. High Energy Phys. 09 (2016) 129.

[17] J. E. Camargo-Molina, A. P. Morais, A. Ordell, R. Pasechnik, M. O. P. Sampaio, and J. Wessén, Reviving trinification models through an $E_{6}$-extended supersymmetric GUT, Phys. Rev. D 95, 075031 (2017).

[18] J. E. Camargo-Molina, A. P. Morais, A. Ordell, R. Pasechnik, and J. Wessén, Scale hierarchies, symmetry breaking and particle spectra in SU(3)-family extended SUSY trinification, Phys. Rev. D 99, 035041 (2019).

[19] A. P. Morais, R. Pasechnik, and W. Porod, Prospects for new physics from gauge left-right-colour-family grand unification, arXiv:2001.06383.

[20] A. P. Morais, R. Pasechnik, and W. Porod, Grand Unified origin of gauge interactions and families replication in the Standard Model, arXiv:2001.04804.

[21] W. Grimus and H. Kuhbock, Fermion masses and mixings in a renormalizable $\mathrm{SO}(10) \times \mathrm{Z}(2)$ GUT, Phys. Lett. B 643, 182 (2006).

[22] F. Bazzocchi, M. Frigerio, and S. Morisi, Fermion masses and mixing in models with $\mathrm{SO}(10) \times \mathrm{A}(4)$ symmetry, Phys. Rev. D 78, 116018 (2008).

[23] C. Hagedorn, M. A. Schmidt, and A. Yu. Smirnov, Lepton mixing and cancellation of the Dirac mass hierarchy in SO(10) GUTs with flavor symmetries T(7) and Sigma(81), Phys. Rev. D 79, 036002 (2009).

[24] W. Grimus and H. Kuhbock, Embedding the ZeeWolfenstein neutrino mass matrix in an $\mathrm{SO}(10) \times \mathrm{A}(4)$ GUT scenario, Phys. Rev. D 77, 055008 (2008). 
[25] A. Albaid, Fermion masses and mixings in a minimal $\mathrm{SO}(10) \times \mathrm{A}(4)$ SUSY GUT, Phys. Rev. D 80, 093002 (2009).

[26] S. F. King and C. Luhn, A new family symmetry for SO(10) GUTs, Nucl. Phys. B820, 269 (2009).

[27] S. F. King and C. Luhn, A supersymmetric grand unified theory of flavour with $\operatorname{PSL}(2)(7) \times \mathrm{SO}(10)$, Nucl. Phys. B832, 414 (2010).

[28] B. Dutta, Y. Mimura, and R. N. Mohapatra, An SO(10) grand unified theory of flavor, J. High Energy Phys. 05 (2010) 034.

[29] A. S. Joshipura and K. M. Patel, Quasi-degenerate neutrinos in SO(10), Phys. Rev. D 82, 031701 (2010).

[30] K. M. Patel, An $S O(10) \times S_{4}$ model of quark-lepton complementarity, Phys. Lett. B 695, 225 (2011).

[31] P.S. Bhupal Dev, B. Dutta, R. N. Mohapatra, and M. Severson, $\theta_{13}$ and proton decay in a minimal $S O(10) \times$ $S_{4}$ model of flavor, Phys. Rev. D 86, 035002 (2012).

[32] P. M. Ferreira, W. Grimus, D. Jurčiukonis, and L. Lavoura, Flavour symmetries in a renormalizable $\mathrm{SO}(10)$ model, Nucl. Phys. B906, 289 (2016).

[33] I. P. Ivanov and L. Lavoura, SO(10) models with flavour symmetries: Classification and examples, J. Phys. G 43, 105005 (2016).

[34] B. Bajc and A. Yu. Smirnov, Hidden flavor symmetries of SO(10) GUT, Nucl. Phys. B909, 954 (2016).

[35] F. Björkeroth, F. J. de Anda, S. F. King, and E. Perdomo, A natural $\mathrm{S}_{4} \times \mathrm{SO}(10)$ model of flavour, J. High Energy Phys. 10 (2017) 148.

[36] F. J. de Anda, S. F. King, and E. Perdomo, $\mathrm{SO}(10) \times \mathrm{S}_{4}$ grand unified theory of flavour and leptogenesis, J. High Energy Phys. 12 (2017) 075.

[37] S. F. King, Unified models of neutrinos, flavour and $C P$ violation, Prog. Part. Nucl. Phys. 94, 217 (2017).

[38] P. B. Pal, Representation-independent manipulations with Dirac spinors, arXiv:physics/0703214.

[39] T. Bandyopadhyay, B. Brahmachari, and A. Raychaudhuri, Implications of the CMS search for $\mathrm{W}_{R}$ on grand unification, J. High Energy Phys. 02 (2016) 023.
[40] J. C. Pati and A. Salam, Lepton number as the fourth color, Phys. Rev. D 10, 275 (1974).

[41] E. Witten, Neutrino masses in the minimal O(10) theory, Phys. Lett. 91B, 81 (1980).

[42] F. del Aguila and L. E. Ibañez, Higgs bosons in SO(10) and partial unification, Nucl. Phys. B177, 60 (1981).

[43] R. N. Mohapatra and G. Senjanović, Higgs Boson effects in grand unified theories, Phys. Rev. D 27, 1601 (1983).

[44] M. Tanabashi et al. (Particle Data Group Collaboration), Review of particle physics, Phys. Rev. D 98, 030001 (2018).

[45] B. Holdom, Two U(1)'s and epsilon charge shifts, Phys. Lett. 166B, 196 (1986).

[46] F. del Aguila, M. Masip, and M. Perez-Victoria, Physical parameters and renormalization of $U(1)_{a} \times U(1)_{b}$ models, Nucl. Phys. B456, 531 (1995).

[47] M.-x. Luo and Y. Xiao, Renormalization group equations in gauge theories with multiple U(1) groups, Phys. Lett. B 555, 279 (2003).

[48] S. Rajpoot, Symmetry breaking and intermediate mass scales in the $\mathrm{SO}(10)$ grand unified theory, Phys. Rev. D 22, 2244 (1980).

[49] M. Fukugita and T. Yanagida, Physics of Neutrinos and Applications to Astrophysics (Springer, New York, 2003).

[50] D. Meloni, T. Ohlsson, and M. Pernow, Threshold effects in $\mathrm{SO}(10)$ models with one intermediate breaking scale, arXiv:1911.11411.

[51] F. Gonzalez Canales, A. Mondragon, U. J. S. Salazar, and L. Velasco-Sevilla, $S_{3}$ as a unified family theory for quarks and leptons, J. Phys. Conf. Ser. 485, 012063 (2014).

[52] F. González Canales, A. Mondragón, M. Mondragón, U. J. Saldaña Salazar, and L. Velasco-Sevilla, Quark sector of $S_{3}$ models: Classification and comparison with experimental data, Phys. Rev. D 88, 096004 (2013).

[53] D. Das, U. K. Dey, and P. B. Pal, $S_{3}$ symmetry and the quark mixing matrix, Phys. Lett. B 753, 315 (2016).

[54] D. Cogollo and J. P. Silva, Two Higgs doublet models with an $S_{3}$ symmetry, Phys. Rev. D 93, 095024 (2016). 